\section{Gleichaltrige Männer sind gut für die Frauen}

\author{
Bei Männern ist das relative Sterberisiko umso geringer, je größer der \\ Altersunterschied zur jüngeren Partnerin ist. Frauen haben dagegen eine \\ kürzere Lebenserwartung wenn ihr Partner jünger als sie selbst ist.
}

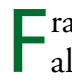
rauen sollten möglichst einen gleichaltrigen Mann heiraten. Ihre Aussichten für ein langes Leben sind dann nämlich am besten. Anders bei Männern: Sie leben umso länger, je jünger ihre Partnerin ist. Dass ein jüngerer Partner für Frauen eher lebensverkür- zend ist, hat eine Studie des Max-PlanckInstituts für demografische Forschung in Rostock festgestellt: Frauen, die einen sieben bis neun Jahre jüngeren Mann heiraten, haben ein um $20 \%$ höheres Mortalitätsrisiko. Die Autoren vermuten, dass Paare, bei denen der Mann jünger ist, damit gegen soziale Normen verstoßen. Dies wiederum könne soziale Sanktionen nach sich ziehen, die gesundheitsschädigenden Stress erzeugen. Unabhängig davon bleibt die Tatsache bestehen, dass verheirateteMänner und Frauen im Schnitt länger leben als unverheiratete.

red

Drefahl S. SHow Does the Age Gap Between Partners Affect Their Survival?

Demography 2010; 47:313-26

\section{Raynaud und Neuropathie nach Chemotherapie}

\begin{abstract}
Neurologische Nebenwirkungen nach Cisplatin-basierter Chemotherapie sind bekannt. An einer großen Kohorte langzeitig überlebender Hodentumor-Patienten in Norwegen wurde geprüft, wie häufig Raynaud-ähnliche Phänomene und neurologische Symptome tatsächlich sind.
\end{abstract}

nsgesamt 1.814 Männer, die zwischen 1980 und 1994 wegen eines einseitigen malignen Hodentumors behandelt und im norwegischen Krebsregister erfasst worden waren, wurden zwischen 1998 und 2002 zu einer Follow-up-Studie eingeladen. 1.409 beantworteten den per Post zugesendeten Fragebogen, der die „six item scale for chemotherapy-induced neurotoxicity“ (SCIN) enthielt, und/oder nahmen an einer audiometrischen Prüfung teil. Von ihnen hatten 528 eine Chemotherapie mit/ohne nachfolgende Strahlentherapie und mit/ohne retroperitoneale Lymphknotendissektion erhalten. 425 wurden mit 1-4 Zyklen, 46 mit $\geq 5$ Zyklen und 57 mit dosisintensivierter Chemotherapie behandelt. Standard war initial entweder Cisplatin in Kombination mit Vinblastin und Bleomycin (CVB, $\mathrm{n}=202)$, mit Bleomycin und Etoposid (BEP, $\mathrm{n}=287$ ) oder mit Etoposid $(n=9)$. Die Standarddosis von Cisplatin betrug $20 \mathrm{mg} / \mathrm{m}^{2}$ über fünf konsekutive Tage, die dosisintensivierte Therapie $100 \mathrm{mg} / \mathrm{m}^{2}$ über zwei konsekutive Tage beziehungsweise insgesamt $>100 \mathrm{mg} / \mathrm{m}^{2}$. 609 Patienten erhielten nur Strahlentherapie, 153 lediglich eine retroperitoneale Lymphknotendissektion, 119 wurden überwacht.
Die mediane Zeit von der Orchiektomie bis zur Beantwortung des Fragebogens betrug 10,7 Jahre. Alle chemotherapierten Patienten hatten statistisch signifikant höhere Risiken für schwerere neurologische Nebenwirkungen und schlechtere audiometrische Ergebnisse als Patienten ohne Chemotherapie: So berichteten 39 vs. $12 \%$ über beträchtliche oder schwere Raynaud-ähnliche Phänomene (weiße oder kalte Hände/Finger oder Füße/Zehen) bei Kälteexposition. Bei 21 vs. $12 \%$ war das Hörvermögen schlecht. 22 vs. $12 \%$ berichteten über Tinnitus als Hauptproblem von beträchtlichem oder schwerem Ausmaß. Hörverschlechterung und Tinnitus waren bei dosisintensivierter Therapie häufiger als bei keiner Chemotherapie (Odds Ratio 5,3 bzw. 7,1).

Die Audiometrie bei $4.000 \mathrm{~Hz}$ ergab, dass signifikant mehr Männer mit als ohne Chemotherapie in der Quartile mit der stärksten Hörverschlechterung waren (28\% bei $1-4$ Zyklen; $37 \%$ bei $\geq 5 \mathrm{Zy-}$ klen; $69 \%$ bei dosisintensivierter Therapie). Nach Strahlentherapie waren Parästhesien der Füße signifikant häufiger (Odds Ratio 1,5).

Fazit: Langzeit-Überlebende nach Cisplatin-basierter Chemotherapie wegen Hodenkrebs leiden häufiger unter dosisabhängigen neurologischen Nebenwirkungen und Raynaud-ähnlichen Phänomenen als solche ohne Chemotherapie.

Brydøy $M$ et al. Observational study of prevalence of long-term Raynaud-like phenomena and neurological side effects in testicular cancer survivors.

J Natl Cancer Inst 2009; 101: 1682-95.

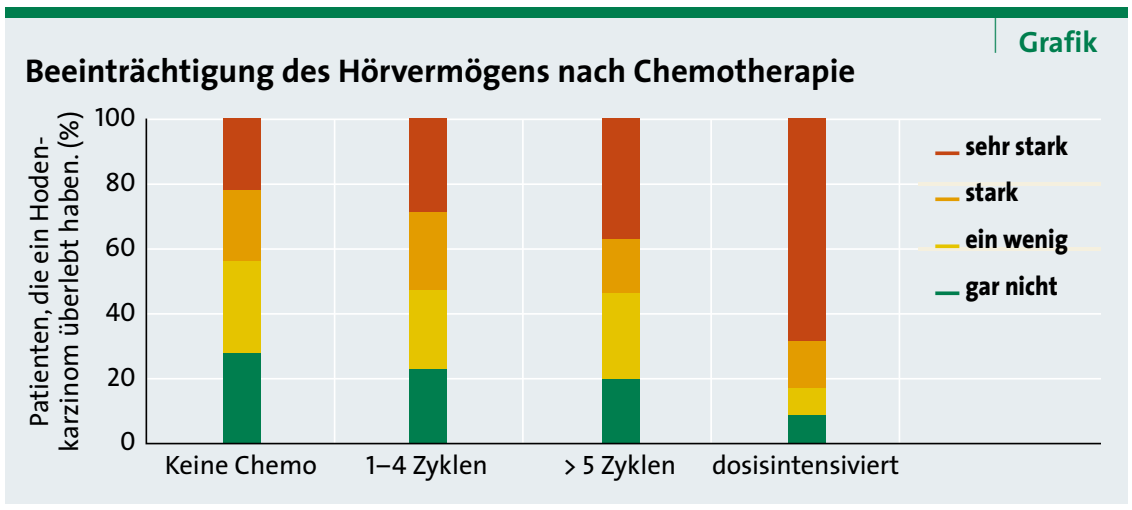

